\title{
Gestão de micro e pequenas empresas na Engenharia de Produção: revisão
}

\section{sistemática de literatura}

\author{
Micro and small business management in Production Engineering: systematic literature review \\ Gestión de micro y pequeñas empresas en Ingeniería de Producción: revisión sistemática de la
}

literatura

Recebido: 30/09/2021 | Revisado: 09/10/2021 | Aceito: 15/10/2021 | Publicado: 17/10/2021

\author{
Thairone Ezequiel de Almeida \\ ORCID: https://orcid.org/0000-0002-0776-544X \\ Universidade Federal de Ouro Preto, Brasil \\ E-mail: thairone.almeida@aluno.ufop.edu.br \\ Isabela Carvalho de Morais \\ ORCID: https://orcid.org/0000-0001-8274-406X \\ Universidade Federal de Ouro Preto, Brasil \\ E-mail: isabela.morais@ufop.edu.br \\ Luciana Paula Reis \\ ORCID: https://orcid.org/0000-0002-6009-8674 \\ Universidade Federal de Ouro Preto, Brasil \\ E-mail: lucianapaula@ufop.edu.br
}

\begin{abstract}
Resumo
A maior parte dos cursos de graduação em Engenharia de Produção apresenta projetos pedagógicos modernos e em concordância com as Diretrizes Curriculares Nacionais de Engenharia. Contudo, a criação de estratégias voltada ao universo da Gestão de Micro e Pequenas Empresas (GMPE) tem atributos nem sempre revelados nos cursos. O elemento central deste artigo é identificar quais vertentes existem na literatura quanto ao ensino-aprendizagem de GMPE nos cursos de graduação em Engenharia de Produção. A Revisão Sistemática de Literatura (RSL) resultou em 20 artigos publicados entre 2016-2021, obtidos na base de dados do Portal de Periódicos da Coordenação de Aperfeiçoamento de Pessoal de Nível Superior (CAPES). Percebe-se na revisão que o tema foi tratado por intermédio das dimensões de especificidades de GMPE; estrutura curricular para formação do engenheiro de produção; e ensinoaprendizagem do empreendedorismo. Destaca-se a escassez de publicações que tratam das particularidades de criação de estratégias voltadas às Micro e Pequenas Empresas (MPE's) nos Projetos Pedagógicos dos Cursos (PPC's) de Engenharia de Produção. Por ser um assunto atual e emergente, o estudo proposto pode contribuir para a compreensão do GMPE por meio de tais cursos e gerar revelações que nortearão pesquisas futuras.

Palavras-chave: Educação em Engenharia de Produção; Diretrizes curriculares dos cursos de engenharia; Projeto pedagógico do curso; Especificidades das micro e pequenas empresas; Gestão de micro e pequenas empresas.
\end{abstract}

\footnotetext{
Abstract

Most undergraduate courses in Production Engineering have modern pedagogical projects following the national engineering curriculum guidelines. However, the creation of strategies aimed at the universe of Micro and Small Business Management (MSBM) has attributes that are not always clear in the courses. This article's central element is to identify which strands exist in the literature regarding the teaching-learning of the MSBM in undergraduate courses in Production Engineering. The systematic literature review resulted in 20 articles published between 2016-2021, obtained from the Coordination for the Improvement of Higher Education Personnel (CAPES)'s database. The literature review addressed the topic through the dimensions of specificities of MSBM, curriculum structure for training in the Production Engineering course, and teaching-learning of entrepreneurship. The literature review also highlights the scarcity of publications that deal with the strategies aimed at micro and small enterprises in the Pedagogical Projects of Production Engineering Courses. The proposed study can contribute to the understanding of MSBM by such courses and generate revelations that guide future research.

Keywords: Education in Production Engineering; Curriculum guidelines for engineering courses; Course pedagogical project; Specifics of micro and small businesses; Micro and small business management.

\section{Resumen}

La mayoría de los cursos de pregrado en Ingeniería de Producción presentan proyectos pedagógicos modernos y de acuerdo con los lineamientos del currículo nacional de ingeniería. Sin embargo, la creación de estrategias dirigidas al universo de la Gestión de la Micro y Pequeña Empresa (GMPE) tiene atributos que no siempre se revelan en los
} 
cursos. El elemento central del artículo es identificar qué vertientes de la literatura existen sobre la enseñanzaaprendizaje de la GMPE en los cursos de pregrado en Ingeniería de Producción. La revisión sistemática de la literatura resultó en 20 artículos publicados entre 2016-2021, obtenidos de la base de datos del Portal de Revistas de la Coordinación para el Perfeccionamiento del Personal de Educación Superior (CAPES). Se puede observar en la revisión que el tema se abordó a través de las dimensiones de especificidades de la GMPE; estructura curricular para la formación del ingeniero de producción; y enseñanza-aprendizaje del espíritu empresarial. Se destaca la escasez de publicaciones que aborden las particularidades de la creación de estrategias dirigidas a la micro y pequeñas empresas en los proyectos pedagógicos. Al ser un tema actual y emergente, el estudio propuesto puede contribuir a la comprensión de la GMPE a través de dichos cursos y generar revelaciones que orientarán futuras investigaciones.

Palabras clave: Educación en Ingeniería de Producción; Directrices curriculares para cursos de ingeniería; Proyecto pedagógico del curso; Específicos de micro y pequeñas empresas; Gestión de micro y pequeñas empresas.

\section{Introduçãa}

Crescentemente percebe-se a necessidade da atuação do engenheiro de produção com perfil qualificado e capaz de transferir o conhecimento, além de compreender os processos produtivos, supervisionar pessoas e estar disposto a enfrentar novos desafios (Azevedo \& Gontijo, 2017). Em seu documento relativo à grande área e diretrizes curriculares, a Associação Brasileira de Engenharia de Produção (ABEPRO, 1998, p. 1) afirma que "compete à engenharia de produção o projeto, a modelagem, a implantação, a operação, a manutenção e a melhoria de sistemas produtivos integrados de bens e serviços, envolvendo homens, recursos financeiros e materiais, tecnologia, informação e energia".

A maior parte dos cursos de graduação em Engenharia de Produção denota projetos pedagógicos modernos e em concordância com as Diretrizes Curriculares Nacionais dos Cursos de Graduação em Engenharia da Câmara de Educação Superior (CES) do Conselho Nacional de Educação (CNE) do Ministério da Educação (MEC) (Brasil, 2019). As disciplinas são compatíveis à formação do profissional para exercer atividade em empresas de diferentes portes e tamanhos.

Apesar disso, conforme Crnkovic e Moretti (2012), a gestão de negócios voltada ao universo das Micro e Pequenas Empresas (MPE’s) tem atributos nem sempre revelados nos cursos. Muitos engenheiros de produção que são proprietários ou atuam em empresas de pequeno porte não foram estimulados durante a graduação a inovarem, agirem com celeridade, preverem crises, perceberem tendências e criarem novas oportunidades em imprevistos do cotidiano organizacional (Delgado et al., 2018).

Empresas com tamanhos distintos apresentam diferentes desafios, que demandam soluções peculiares. Pesquisas afirmam que as MPE's se diferenciam das grandes empresas por figurarem uma gestão centralizada e estrutura organizacional com poucos níveis hierárquicos. Isso implica na atuação direta e empirismo do gestor/proprietário na relação com os clientes, gestão de pessoas pouco profissionalizada, sistemas de informação simples, produção em pequena escala e recursos escassos. A tomada de decisão é intuitiva e, em sua maioria, baseada nas atividades de curto prazo (Almeida, 2020; Leone, 1991, 1999; Leone \& Leone, 2012; Martins, Leone \& Leone, 2017).

Nesse âmbito, a demanda de propostas pedagógicas que visam a promoção de estratégias de ensino-aprendizagem voltadas ao gerenciamento de empresas de pequeno porte indica a escassez de estudos que determinam elementos significativos para os conteúdos programáticos aptos a capacitar os discentes dos cursos de graduação de Engenharia de Produção a tomarem decisões como parte complementar da organização (Almeida, 2019). Assim, há um campo de pesquisa ainda pouco explorado na educação em engenharia de produção, a Gestão de Micro e Pequenas Empresas (GMPE).

O elemento central deste artigo é identificar quais vertentes existem na literatura quanto ao ensino-aprendizagem de GMPE nos cursos de graduação em Engenharia de Produção. Para atingir tal objetivo, faz-se necessário nesta pesquisa mapear a literatura sobre o tema e organizar os artigos encontrados em vertentes abordadas na bibliografia recente. Essas vertentes estão relacionadas à GMPE, no entanto mostram uma lacuna para pesquisas específicas sobre o tema. Na sequência, serão expostos os procedimentos metodológicos utilizados na Revisão Sistemática de Literatura (RSL), os resultados e a discussão. 


\section{Procedimentos Metodológicos}

A RSL pode ser entendida como uma pesquisa que utiliza dados da literatura como fonte para o estudo de determinado tema. O ponto de partida para a revisão foi a leitura de duas importantes obras: Escrivão Filho (2006) e Crnkovic e Moretti (2012). A partir do reconhecimento dos textos, foi necessário formular a pergunta da situação-problema para definir a estrutura da revisão, a saber: Quais vertentes existem na literatura quanto ao ensino-aprendizagem de GMPE nos cursos de graduação em Engenharia de Produção?

Com base em Sampaio e Mancini (2007), foi definida a estratégia de busca de outras obras que consiste em oito etapas: (1) buscar artigos nas bases de dados por intermédio das combinações de palavras-chave; (2) identificar artigos por meio dos títulos e resumos; (3) recuperar artigos; (4) selecionar os estudos primários de acordo com os critérios de inclusão e exclusão; (5) extrair os dados; (6) avaliar a qualidade; (7) realizar síntese; e (8) redigir resultados e interpretar.

Para fazer a busca nas bases de dados mediante as combinações de palavras-chave, foram utilizados alguns descritores da área de estudo, em português e inglês, a saber: "conteúdo programático (programmatic content/syllabus)"; "currículo de engenharia de produção (production engineering curriculum/industrial engineering curriculum/industrial engineering programs)"; "diretrizes curriculares para os cursos de engenharia (curricular guidelines for engineering courses)"; "educação em engenharia de produção (education production engineering/education industrial engineering)"; "gestão de micro e pequenas empresas (micro and small enterprises management/micro and small business management)"; "gestão de microempresas (microenterprise management)"; "gestão de pequenas empresas (small business management)"; e "micro e pequenas empresas (micro and small enterprises)".

As bases de dados utilizadas para processo de busca foram Google Scholar, Portal de Periódicos da Coordenação de Aperfeiçoamento de Pessoal de Nível Superior (CAPES), Scientific Electronic Library Online (SciELO) e Scopus. A pesquisa seguiu os critérios de inclusão (a) recorte temporal de 2016 a 2021; (b) estudo disponível em texto completo; (c) estudo disponível no idioma português, inglês ou espanhol; (d) estudo que aborda a temática; (e) maior quantidade de resultados gerados pelas combinações de palavras-chave; e (f) estudo ser um artigo científico. Como critérios de exclusão foram desconsiderados os estudos que (i) não respondiam a situação-problema orientadora da pesquisa; (ii) apresentaram duplicatas em mais de uma base de dados; e (iii) não pertenciam a periódicos reconhecidos na área de engenharia de produção.

$\mathrm{Na}$ investigação, os termos foram colocados entre aspas (exibição de documentos que possuam em seu conteúdo exatamente a frase em questão) e combinados pelo operador booleano “and”. Não foram considerados os estudos identificados sem combinações de descritores (palavra-chave/keyword unitária). Em um primeiro momento, foi aplicado o critério de inclusão (a) e identificados 344 estudos com combinações de descritores em português (337 da base de dados Google Scholar e sete da CAPES); e 681 estudos com combinações de descritores em inglês (662 da base de dados Google Scholar e 19 da CAPES). As bases de dados SciELO e Scopus não retornaram nenhum estudo. Logo depois, os critérios de inclusão (b), (c), (d), (e) e (f) e os de exclusão (i), (ii) e (iii) mostraram que somente a base do Portal de Periódicos da CAPES possuía artigos relevantes para a RSL. Todos os artigos existentes na CAPES estavam presentes na base de dados Google Scholar (duplicatas). Após a seleção criteriosa, foram identificados um artigo no idioma português, 18 em inglês e um em espanhol. A Figura 1 ilustra as etapas para a seleção dos artigos nas bases de dados. 
Figura 1 - Seleção de artigos nas bases de dados.

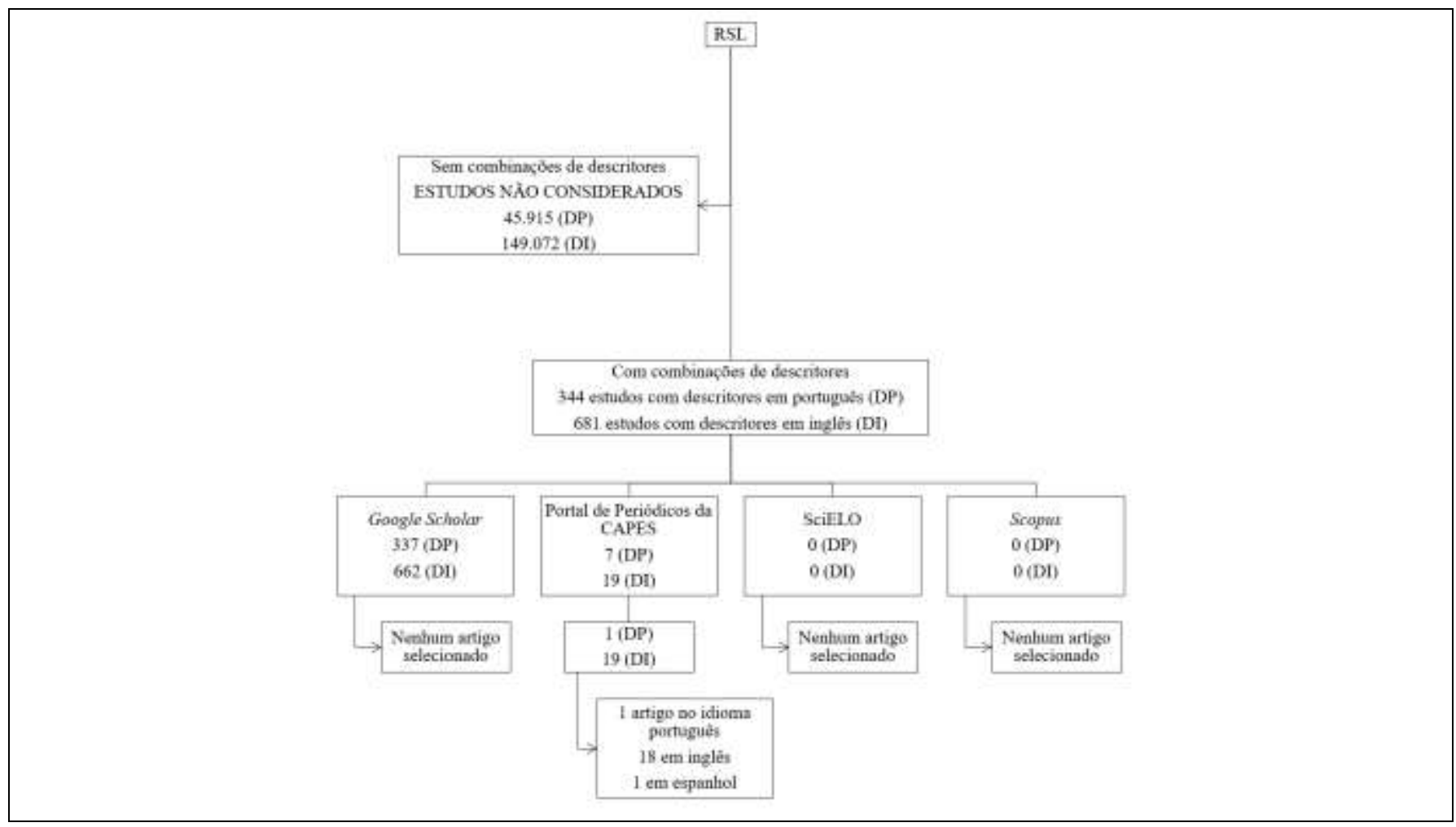

Fonte: Autores.

Os artigos foram nomeados por meio da combinação de palavras-chave/autoria/ano e recuperados no gerenciador de referências Mendeley. Para a extração dos dados, foi feita a leitura dos trabalhos e todos foram considerados aptos para a RSL, em função do seu grau de detalhamento, dos autores em ordem alfabética, a saber: Almeida (2019); Balza-Franco (2016); Bandera et al. (2018); Franchetti e Ariss (2017); Gamede e Uleanya (2019); Gargouri e Naatus (2019); Ghina, Simatupang e Gustomo (2017); Glackin, Byrd e Phelan (2016); Jones e Liu (2017); Kolvereid e Iermolenko (2020); Ladeveze e Canal (2016); Marliyah (2018); Michael (2018); Nguyen e Nguyen (2018); Ramona-Diana (2017); Reinert e Gontijo (2017); Saji e Nair (2018); Salah et al. (2020); Santoso (2017); e Sun et al. (2017).

Imediatamente, na intenção de verificar como o tema foi discutido na esfera acadêmica, foram feitas a síntese, a redação e a interpretação das principais ideias dos textos. Como resultado, foi redigida a seção da discussão do artigo. O período de realização da RSL foi de setembro/2020 a junho/2021. Os procedimentos metodológicos realizados nesta investigação estão representados na Figura 2, que proporciona um melhor entendimento da RSL.

Figura 2 - Condução da RSL.

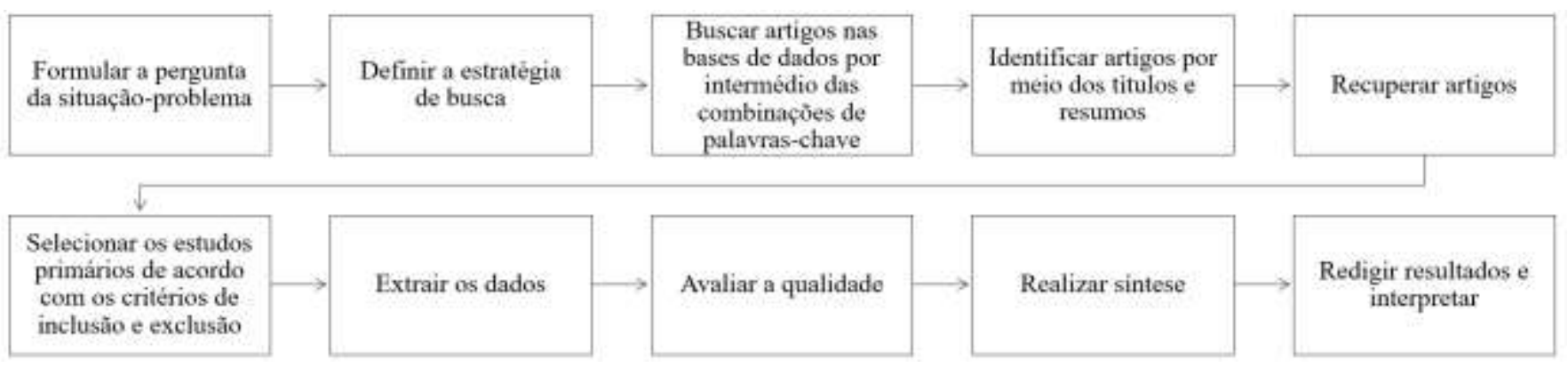

Fonte: Adaptado de Sampaio e Mancini (2007). 


\section{Resultados}

Diante dos achados, três (15\%) dos artigos são do ano de 2016, sete (35\%) são do ano de 2017, cinco (25\%) são do ano de 2018, três (15\%) são do ano de 2019 e dois (10\%) são do ano de 2020. Com relação à dimensão de citações recebidas, o trabalho de Sun et al. (2017), que aborda o impacto da educação empreendedora na intenção empreendedora de estudantes de engenharia em Hong Kong, foi o que obteve o maior número de referências na literatura (41 citações). Em ordem decrescente, a Tabela 1 mostra a quantidade de citações de cada artigo.

Tabela 1 - Quantidade de citações por artigo (junho/2021).

\begin{tabular}{|c|c|}
\hline $\begin{array}{c}\text { Artigo } \\
\text { (autores) }\end{array}$ & Quantidade de citações \\
\hline Sun et al. (2017) & 41 \\
\hline Ghina, Simatupang e Gustomo (2017) & 28 \\
\hline Bandera et al. (2018) & 16 \\
\hline Jones e Liu (2017) & 15 \\
\hline Ramona-Diana (2017) & 15 \\
\hline Balza-Franco (2016) & 13 \\
\hline Franchetti e Ariss (2017) & 8 \\
\hline Gamede e Uleanya (2019) & 8 \\
\hline Ladeveze e Canal (2016) & 7 \\
\hline Marliyah (2018) & 6 \\
\hline Reinert e Gontijo (2017) & 5 \\
\hline Michael (2018) & 4 \\
\hline Nguyen e Nguyen (2018) & 4 \\
\hline Glackin, Byrd e Phelan (2016) & 3 \\
\hline Santoso (2017) & 3 \\
\hline Gargouri e Naatus (2019) & 2 \\
\hline Salah et al. (2020) & 2 \\
\hline Almeida (2019) & 0 \\
\hline
\end{tabular}

Fonte: Autores.

Dos periódicos utilizados na RSL, o que deteve mais publicações foi o Journal of Entrepreneurship Education, com cinco artigos. Os outros 15 periódicos possuem um artigo cada, a saber: Academy of Entrepreneurship Journal; Academy of Strategic Management Journal; Benchmarking: An International Journal; European Journal of Training and Development; Gestão da Produção, Operações e Sistemas (GEPROS); International Journal of Information, Business and Management; Journal of Engineering Design; Journal of Entrepreneurship, Management and Innovation; Journal of Information Systems Education; Journal of Small Business Management; International Journal of STEM Education; Management Decision; Processes; Revista de la Educación Superior; e Revista Latina de Comunicación Social.

Descrita as características básicas dos artigos e periódicos que compõem a RSL, a seguir serão relatadas as contribuições dos estudos no intuito de assimilar de forma ampla, quais vertentes existem na literatura quanto ao ensinoaprendizagem de GMPE nos cursos de graduação em Engenharia de Produção. A fim de facilitar o entendimento da RSL, tais contribuições serão discutidas na próxima seção.

\section{Discussão}

O processo de ensino-aprendizagem pode ser estabelecido como a transferência de conhecimento entre docentes e discentes, no qual o educando e o educador ensinam e aprendem um com o outro (Ten Caten, 2020). O debate dessa temática associada à GMPE na engenharia de produção não é uma preocupação recente. É importante referir que parte dos profissionais da engenharia de produção, ao finalizar os cursos, não vão trabalhar em empresas de grande porte, pois preferem criar o seu próprio negócio. O dilema, nesta situação, é que gerenciar uma MPE é diferente de gerenciar uma grande. E muitas vezes, as 
estruturas curriculares desses cursos não abordam a temática (Crnkovic \& Moretti, 2012).

A partir da RSL, observou-se que os estudos recentes que investigam o tema abordam três vertentes relacionadas aos resultados obtidos das combinações de palavras-chaves: (1) dimensões de especificidades de GMPE; (2) estrutura curricular para formação do engenheiro de produção; e (3) ensino-aprendizagem do empreendedorismo. Essas foram as principais vertentes encontradas nos artigos, ou melhor, o achado principal. Elas foram definidas com base no mapeamento da literatura $\mathrm{e}$ organização dos artigos. Vale frisar que a maioria dos artigos não aborda especificamente a GMPE, mas sim alguns elementos dela, como por exemplo, o empreendedorismo. A Figura 3 relaciona as vertentes da RSL e os autores.

Figura 3 - Vertentes da RSL

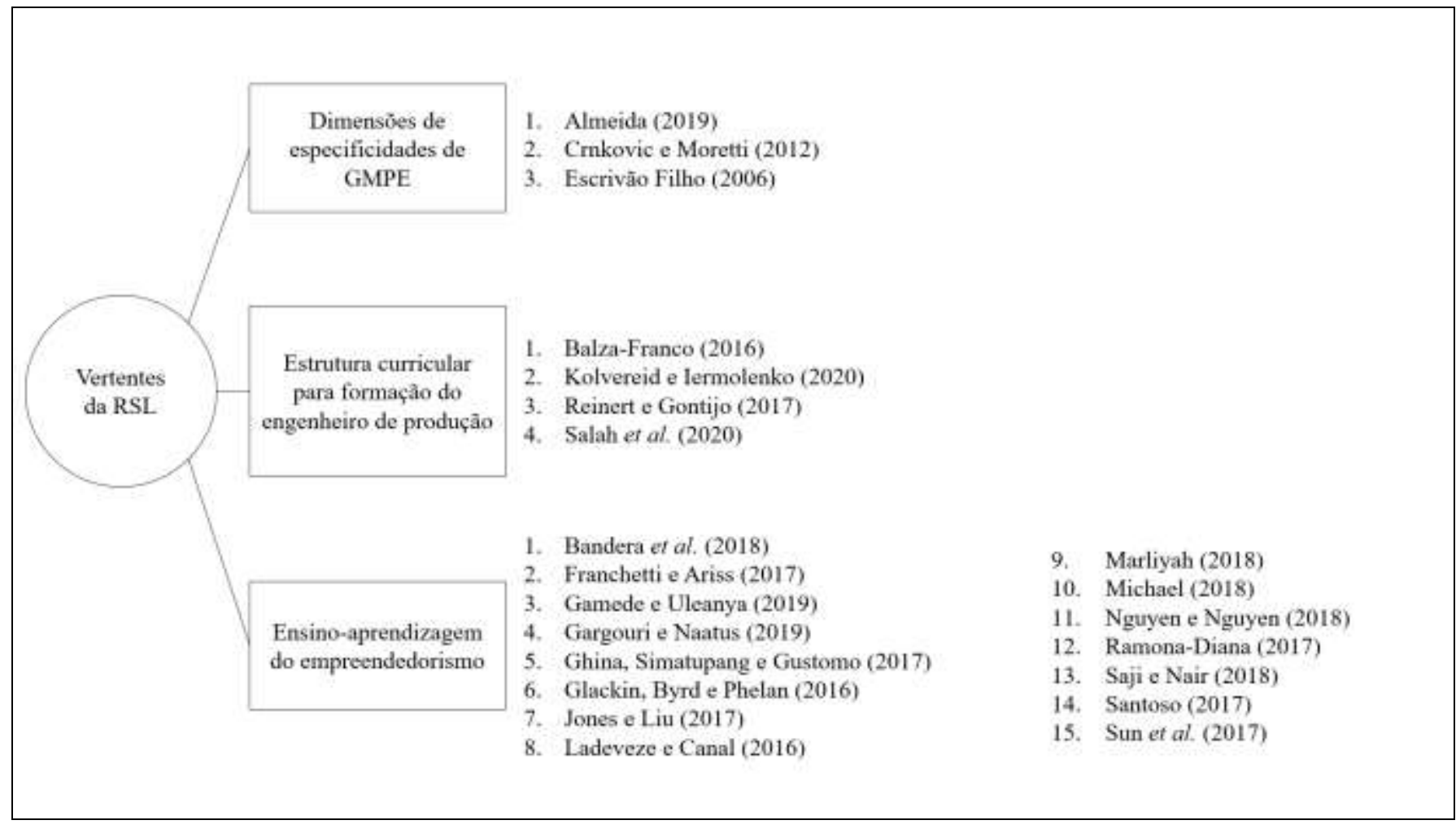

Fonte: Autores.

Na primeira vertente, em sua tese de livre-docência sobre o reconhecimento das especificidades organizacionais da pequena empresa, Escrivão Filho (2006) busca compreender, por meio da composição de um mapa organizacional, a atuação de fatores restritivos e facilitadores da administração estratégica. Para o autor, são três as dimensões que, relacionadas as escolas prescritivas e descritivas, influenciam na criação de estratégias para gerir a MPE, a saber: dirigente (fundamentado no conceito de liderança); organização (fundamentado na estruturação de recursos e competências); e ambiente (fundamentado nas oportunidades e forças competitivas). Os resultados sugerem que: (a) a empresa de pequeno porte tem características organizacionais diferentes da grande empresa; e (b) o mapa organizacional é uma ferramenta apta a evidenciar os erros da administração estratégica na MPE, pois aponta incoerências entre os três componentes quando coordenado de modo inadequado.

Em outra investigação, Crnkovic e Moretti (2012) rastreiam itens significativos para a proposta do conteúdo programático da disciplina GMPE. O estudo exploratório foi realizado mediante as Diretrizes Curriculares dos Cursos de Engenharia, das competências e habilidades do engenheiro de produção, do referencial teórico de características de empresas de pequeno porte, e da compreensão das três dimensões de especificidades de criação de estratégias em MPE (dirigente, 
organização e ambiente). Os resultados da pesquisa indicam que: (a) entender como a MPE é gerada, concebida e administrada pode ser um diferencial competitivo para o engenheiro de produção que exercer a profissão na área; (b) ao lecionar uma disciplina voltada à GMPE, deve-se considerar as particularidades relacionadas aos pilares dirigente, organização e ambiente; e (c) a concepção da proposta para o curso de Engenharia de Produção expõe atributos absolutamente diferentes das disciplinas de Empreendedorismo. Isso pois, a bibliografia de GMPE é orientada à administração de negócios que já estão no mercado e procura contemplar características específicas de empresas de pequeno porte que as distinguem das grandes.

Seguindo essa mesma linha de pesquisa, Almeida (2019) realiza um exame sistemático dos principais itens existentes na literatura para elaborar uma proposta pedagógica no desenvolvimento da disciplina GMPE do curso de Engenharia de Produção. $\mathrm{O}$ autor busca fazer um estudo exploratório nas diretrizes curriculares do curso e na literatura relacionada às dimensões de especificidades de criação de estratégias em gestão de empresas de pequeno porte. Como resultado, foi construído um quadro sinóptico para sistematizar um plano de trabalho docente com enfoque nas particularidades das MPE's no processo de ensino-aprendizagem do discente.

Na segunda vertente da RSL, que trata da estrutura curricular para formação do engenheiro de produção, alguns estudos inserem na formulação do Projeto Pedagógico do Curso (PPC) conteúdos relevantes no processo de ensinoaprendizagem aplicáveis às empresas de qualquer porte, a saber: os atributos, pressupostos, processos e variáveis relevantes nos campos de gestão de operações e logística (Balza-Franco, 2016); a importância dos fatores humanos e ergonomia na área de projeto do produto (Reinert \& Gontijo, 2017); o conceito da revolução industrial digital e Indústria 4.0 (Salah et al., 2020); e a empregabilidade e qualidade de vida no mercado de trabalho (Kolvereid \& Iermolenko, 2020). Tais trabalhos reforçam que o PPC evidencia a importância que as Diretrizes Curriculares têm para a formulação dos cursos de graduação de Engenharia de Produção, tanto em termos de organização, como também no desenvolvimento e na avaliação dos cursos. Mas, os estudos não abordam de forma específica como as estruturas curriculares dos cursos tratam as particularidades na criação de estratégias aplicadas à GMPE.

Finalmente, a terceira vertente mostra que a RSL retornou artigos de ensino-aprendizagem do empreendedorismo alusivo à MPE. Diversos estudos revelam que o mercado de trabalho exige que os educadores entendam e ajustem o currículo às disparidades culturais pertinentes as atitudes, autopercepções, competências, habilidades, valores e intenções dos estudantes universitários em relação ao comportamento organizacional em empresas de pequeno porte (Bandera et al., 2018; Franchetti \& Ariss, 2017; Gamede \& Uleanya, 2019; Gargouri \& Naatus, 2019; Ghina, Simatupang \& Gustomo, 2017; Ladeveze \& Canal, 2016; Nguyen \& Nguyen, 2018; Ramona-Diana, 2017; Saji \& Nair, 2018). Outras investigações ainda abordam o empreendedorismo como forma de apoiar o discente de engenharia de produção - e outros cursos - a exercer a profissão em pequenas empresas, como evidenciado nos artigos de Glackin, Byrd e Phelan (2016), Jones e Liu (2017), Marliyah (2018), Michael (2018), Santoso (2017) e Sun et al. (2017).

Apesar disso, além da autoconsciência, autocontrole, motivação, empatia e habilidades sociais necessárias para empreender (Bianchi, 2019), é preciso identificar, coordenar e integrar as especificidades de criação de estratégias para a GMPE. A estrutura curricular que contempla a temática deve abordar a dinâmica gerencial das empresas de pequeno porte $\mathrm{e}$ ser pautada pelos pilares dirigente, organização e ambiente. Isto posto, o aperfeiçoamento de estratégias orientadas à GMPE ocorre de maneira específica, que vai mais adiante de disciplinas como Empreendedorismo (Crnkovic \& Moretti, 2012); especificidade essa que os discentes dos cursos de graduação em Engenharia de Produção, por vezes, não são incentivados a perceber. 


\section{Considerações Finais}

O objetivo desse artigo foi realizar um exame metódico na bibliografia para identificar quais vertentes existem na literatura quanto ao ensino-aprendizagem de GMPE nos cursos de graduação em Engenharia de Produção. Os procedimentos metodológicos da RSL resultaram em 20 artigos fundamentais para o estudo, em função do grau de detalhamento. Os resultados desta pesquisa são válidos, apesar de não serem genéricos, uma vez que cada instituição de ensino superior pode tratar do ensino-aprendizagem de GMPE em sua estrutura curricular de modo transversal no ensino, pesquisa e extensão.

Por meio da exploração, foi possível perceber o quanto é importante o método da RSL na construção de uma revisão teórica. Considera-se que o aprofundamento do conhecimento sobre o tema discutido permitiu que os autores apurassem pesquisas e extraíssem conceitos e achados acadêmicos que especificam dados para descobrir, em outro trabalho, como os cursos de graduação de Engenharia de Produção abordam o ensino-aprendizagem de GMPE.

Uma limitação importante a ser apresentada, foi a escassez de publicações que tratam justamente da criação de estratégias específicas para a MPE nos PPC's de Engenharia de Produção. Isso dificultou a análise mais profunda de como os cursos tratam da GMPE no processo de ensino-aprendizagem. Percebe-se na revisão que a temática foi tratada pela literatura recente por meio das dimensões de especificidades de GMPE (apenas um artigo); da estrutura curricular para formação do engenheiro de produção (quatro artigos); e do ensino-aprendizagem do empreendedorismo (15 artigos).

As três vertentes encontradas mostram que as estruturas curriculares dos cursos de graduação se preocupam com o ensino-aprendizagem de temas atuais e necessários para várias áreas da Engenharia de Produção, inclusive o empreendedorismo. Mas tais currículos não favorecem a realidade da GMPE. Empreender e/ou criar uma nova organização é diferente de geri-la. Por isso, a utilização de diferentes metodologias e abordagens interdisciplinares e multidisciplinares são fundamentais na aprendizagem de competências transversais.

No que se refere à criação de estratégias, sugere-se que os cursos de graduação em Engenharia de Produção amplifiquem o campo de atuação do profissional, com destaque ao cotidiano organizacional das MPE's - que se difere das grandes. Isso porque, o projeto de uma organização, independente do seu porte e setor de atuação, deve refletir as particularidades de gestão, o perfil do dirigente e o ambiente na qual a empresa está estabelecida. Esse enfoque poderá contribuir na competitividade do engenheiro, além de inserir melhor conhecimento, senso crítico, equilíbrio e discernimento para a atuação em empresas de pequeno porte.

Pesquisas futuras poderão ser desenvolvidas acerca da melhoria ou inclusão de novos itens na RSL, com o intuito de inserir outros componentes da engenharia de produção aplicáveis à realidade do ensino-aprendizagem de GMPE. Por se tratar de um assunto atual e emergente, o estudo proposto pode colaborar com o entendimento de criação de estratégias específicas para MPE's pelos cursos e gerar revelações que orientem outros trabalhos, no sentido de preparar as novas gerações de profissionais para os desafios e tendências do século XXI.

\section{Agradecimentos}

Os autores agradecem à Universidade Federal de Ouro Preto (UFOP), à Pró-reitoria de Pesquisa, Pós-Graduação e Inovação (PROPPI/UFOP), à Fundação de Amparo à Pesquisa do Estado de Minas Gerais (FAPEMIG) e à Coordenação de Aperfeiçoamento de Pessoal de Nível Superior (CAPES) pelo apoio na realização desta pesquisa.

\section{Referências}

ABEPRO (1998). Associação Brasileira de Engenharia de Produção. Engenharia de produção: grande área e diretrizes curriculares. ABEPRO.

Almeida, T. E. (2019). Gestão de micro e pequenas empresas: uma proposta pedagógica para o desenvolvimento de uma disciplina do curso de Engenharia de Produção. Revista Gestão da Produção, Operações e Sistemas (GEPROS), 14(3), 110-121. https://doi.org/10.15675/gepros.v14i3.2609 
Almeida, T. E. (2020). Desafios na gestão logística de uma microempresa fabricante de biscoitos na perspectiva da integração das atividades primárias e de apoio. Research, Society and Development (RSD), 9(8), e722986023. https://doi.org/10.33448/rsd-v9i8.6023

Azevedo, A. A., \& Gontijo, T. S. (2017). Habilidades, competências e o perfil do profissional de engenharia de produção no sudeste brasileiro. Revista Formação Docente, 9(2), 96-109. https://doi.org/10.15601/1332

Balza-Franco, V. (2016). Formulación y diseño de un modelo de vigilancia tecnológica curricular en programas de ingeniería en Colombia. Revista de la Educación Superior, 45(179), 55-77. https://doi.org/10.1016/j.resu.2016.04.008

Bandera, C., Eminet, A., Passerini, K., \& Pon, K. (2018). Using mind maps to distinguish cultural norms between French and United States entrepreneurship students. Journal of Small Business Management, 56(S1), 177-196. https://doi.org/10.1111/jsbm.12398

Bianchi, R. F. (2019). Empreender como escolha e não como necessidade. In Silva, A. L. (Org.), Empreendedorismo universitário (pp. 14-21). Jundiaí: Paco Editorial.

Brasil (2019). Câmara de Educação Superior do Conselho Nacional de Educação do Ministério da Educação. Resolução CNE/CES $n^{o} 2$, de 24 de abril de 2019. Institui as Diretrizes Curriculares Nacionais do Curso de Graduação em Engenharia. http://portal.mec.gov.br/index.php?option=com_docman\&view=download\&alias=112681-rces002-19\&category_slug=abril-2019-pdf\&Itemid=30192

Crnkovic, L. H., \& Moretti, S. L. A. (2012). Gestão de micro e pequenas empresas: uma proposta de disciplina para a formação do engenheiro. Revista Gestão da Produção, Operações e Sistemas (GEPROS), 7(4), 11-24. https://doi.org/10.15675/gepros.v0i4.652

Delgado, V. V., Rodrigues, A. P. A., Castro, C. C., Jacomassi, I., \& Bueno, L. F. (2018). Empreendedorismo e engenharia de produção: o perfil empreendedor do Alumni Unifaj. Revista Intellectus, 49, 114-131. http://www.revistaintellectus.com.br/artigos/51.612.pdf

Escrivão Filho, E. (2006). Pequena empresa e administração estratégica: reconhecendo especificidades, restrições e facilitadores com o mapa organizacional (Tese de livre-docência). Escola de Engenharia de São Carlos, Universidade de São Paulo, São Carlos, SP.

Franchetti, M., \& Ariss, S. (2017). The implementation of senior design capstone projects combining engineering and business students. International Journal of STEM Education, 17(4), 29-34. https://www.learntechlib.org/p/174406/

Gamede, B. T., \& Uleanya, C. (2019). Factors impacting entrepreneurship education in TVET colleges: a case of South Africa. Journal of Entrepreneurship Education, 22(3), 1-12. https://www.abacademies.org/abstract/factors-impacting-entrepreneurship-education-in-tvet-colleges-a-case-of-south-africa-8319.html

Gargouri, C., \& Naatus, M. K. (2019). Building entrepreneurial mindsets in the classroom. Journal of Entrepreneurship Education, 22(3), 1-20. https://www.abacademies.org/articles/building-entrepreneurial-mindsets-in-the-classroom-8232.html

Ghina, A., Simatupang, T. M., \& Gustomo, A. (2017). The relevancy of graduates'competencies to the effectiveness of entrepreneurship education: a case study at SBM ITB-Indonesia. Journal of Entrepreneurship Education, 20(1), 1-24. https://www.abacademies.org/abstract/the-relevancy-of-graduatescompetencies-to-the-effectiveness-of-entrepreneurship-education-a-case-study-at-sbm-itbindone-6570.html

Glackin, C., Byrd, K., \& Phelan, S. (2016). Contextual considerations in entrepreneurial finance education: a systematic analysis of US undergraduate courses. Academy of Entrepreneurship Journal, 22(2), 13-28. https://doi.org/10.13140/RG.2.1.1536.1687

Jones, C. G., \& Liu, D. (2017). Approaches to incorporating IT entrepreneurship into the information systems curriculum. Journal of Information Systems Education, 28(1), 43-57. https://aisel.aisnet.org/jise/vol28/iss1/4

Kolvereid, L., \& Iermolenko, O. (2020). Training Ukrainian military in job creation and job taking. European Journal of Training and Development, 44(4/5), 449-468. https://doi.org/10.1108/EJTD-09-2019-0169

Ladeveze, L. N., \& Canal, M. N. (2016). Notion for entrepreneurship in school entrepreneurial skills training. Revista Latina de Comunicación Social, 71, 1069-1089. https://doi.org/10.4185/RLCS-2016-1135en

Leone, N. M. C. P. G. (1991). A dimensão física das pequenas e médias empresas (PME'S): à procura de um critério homogeneizador. Revista de Administração de Empresas (RAE), 31(2), 53-59. https://doi.org/10.1590/S0034-75901991000200005

Leone, N. M. C. P. G. (1999). As especificidades das pequenas e médias empresas. RAUSP Management Journal, 34(2), 91-94. http://www.spell.org.br/documentos/ver/18123/as-especificidades-das-pequenas-e-medias-empresas/i/pt-br

Leone, R. J. G., \& Leone, N. M. C. P. G. (2012). Pequenas e médias empresas: contribuições para a discussão sobre por que e como medir o seu tamanho. Revista Eletrônica do Mestrado Profissional em Administração da Universidade Potiguar (RAUnP), 4(1), 67-83. https://doi.org/10.21714/raunp.v4i1.194

Marliyah, L. (2018). Model of entrepreneurship education in vocational school on agribusiness study programme. Journal of Entrepreneurship Education, 21(4), 1-9. https://www.abacademies.org/articles/model-of-entrepreneurship-education-in-vocational-school-on-agribusiness-study-programme-7507.html

Martins, J. G. F., Leone, R. J. G., \& Leone, N. M. C. P. G. (2017). Proposta de método para classificação do porte das empresas. Revista Connexio, 6(1), 139155. https://repositorio.unp.br/index.php/connexio/article/view/1679

Michael, S. C. (2018). What should we teach in the new ventures class? An empirical analysis. Journal of Entrepreneurship Education, 21(2), 1-17. https://www.abacademies.org/abstract/what-should-we-teach-in-the-new-ventures-class-an-empirical-analysis-7044.html

Nguyen, A. T., \& Nguyen, N. V. P. (2018). Benchmarking industrial engineering programs. Benchmarking: An International Journal, $25(4), 1194-1212$. https://doi.org/10.1108/BIJ-09-2016-0136

Ramona-Diana, L. (2017). Developing entrepreneurial skills. An educational and intercultural perspective. Journal of Entrepreneurship, Management and Innovation, 13(4), 97-121. https://doi.org/10.7341/20171346 
Research, Society and Development, v. 10, n. 13, e409101321274, 2021

(CC BY 4.0) | ISSN 2525-3409 | DOI: http://dx.doi.org/10.33448/rsd-v10i13.21274

Reinert, F., \& Gontijo, L. A. (2017). The consideration of human factors in product design at the engineering programmes of a Brazilian university. Journal of Engineering Design, 28(10/12), 709-730. https://doi.org/10.1080/09544828.2017.1393656

Saji, B. S., \& Nair, A. R. (2018). Effectiveness of innovation and entrepreneurship education in UAE higher education. Academy of Strategic Management Journal, 17(4), 1-12. https://www.abacademies.org/articles/effectiveness-of-innovation-and-entrepreneurship-education-in-uae-higher-education-7433.html

Salah, B., Khan, S., Ramadan, M., \& Gjeldum, N. (2020). Integrating the concept of Industry 4.0 by teaching methodology in industrial engineering curriculum. Processes, 8(9), 1007-1022. https://doi.org/10.3390/pr8091007

Sampaio, R. F., \& Mancini, M. C. (2007). Systematic review studies: a guide for careful synthesis of the scientific evidence. Brazilian Journal of Physical Therapy, 11(1), 77-82. https://doi.org/10.1590/S1413-35552007000100013

Santoso, S. (2017). Influence of entrepreneurship education, gender, and parent background to relationship between self eficacy and entrepreneurship intention. International Journal of Information, Business and Management, 9(3), 263-274. http://ijibm.site666.com/IJIBM_Vol9No3_Aug2017.pdf\#page=253

Sun, H., Lo, C. T., Liang, B., \& Wong, Y. L. B. (2017). The impact of entrepreneurial education on entrepreneurial intention of engineering students in Hong Kong. Management Decision, 55(7), 1371-1393. DOI: https://doi.org/10.1108/MD-06-2016-0392

Ten Caten, C. S. (2020). Reestruturação do ensino da engenharia de produção: fomentar a inovação e o empreendedorismo. In Santos, J. V. T. (Org.), A universidade do futuro (pp. 133-153). Porto Alegre: Editora da UFRGS. 